\title{
Traumatic Dysgeusia, an Unusual Complication of Facial Trauma: A Case Report
}

\author{
João Paulo Bonardi, DDS, ${ }^{*}$ Fernanda Herrera da Costa, DDS, $\dagger$ \\ Glaykon Alex Vitti Stabile, DDS, MSc, PbD, $\ddagger$ and \\ Cecilia Luiz Pereira-Stabile, DDS, MSc, PhD $\S$
}

\begin{abstract}
The chemical senses of taste and smell are important to human life, because they play an important role in detecting potential environmental hazards. Humans can identify countless different flavors by the simultaneous perception of taste and smell. Reports of sensory loss after craniocerebral trauma began to appear in the medical literature in the middle 1800s. Dysgeusia associated with head injuries is rare and its reported incidence is 0.4 to $0.5 \%$. This report describes the clinical case of a 32-year-old man with Le Fort I and III fractures treated with surgical reduction and fixation. The patient presented with dysgeusia after slight improvement of his preoperative anosmia. The prognosis is favorable and the treatment is prospective.

(C) 2016 American Association of Oral and Maxillofacial Surgeons

J Oral Maxillofac Surg 74:1416-1419, 2016
\end{abstract}

Malfunctions in the chemical senses of taste and smell are often undervalued by lay people and health professionals. However, these senses define food and drink flavors and help in the detection of potential environmental hazards. ${ }^{1}$

Flavor is mediated by specialized neuroepithelial cells grouped into organs called taste buds, which are responsible for detecting a wide range of soluble chemical elements when they are exposed to the tongue. ${ }^{2}$

The variety of flavors might seem immeasurable; however, most researchers classify them into 5 basic groups, namely salty, sour, sweet, bitter, and umami. The innermost part of the tongue is more responsive to sweet, the outermost part is more responsive to bitter, and the sides are more responsive to salty and sour. Humans can perceive countless different flavors by adding taste to smell. ${ }^{3}$

Taste buds in the anterior two thirds of the tongue are innervated by the tympani chorda nerve (a branch of the facial nerve, ie, cranial nerve VII). The glossopharyngeal nerve (cranial nerve IX) innervates the taste buds of the posterior third of the tongue, and special sensory fibers of the vagus nerve (cranial nerve $\mathrm{X}$ ) are responsible for the taste buds of the posterior pharynx. ${ }^{4}$

Metal ions such as nickel $\left(\mathrm{Ni}^{2+}\right)$, zinc $\left(\mathrm{Zn}^{2+}\right)$, and copper $\left(\mathrm{Cu}^{2+}\right)$ can change the detection and recognition levels of taste, because they affect the minimum concentrations at which salt, sugar, urea, and acid are recognized. ${ }^{5}$

Reports describing the loss of smell and taste after craniocerebral trauma began to appear in the medical literature in the middle 1800s. ${ }^{6}$ Dysgeusia (altered taste) associated with head injury is a rare condition, with an incidence of 0.4 to $0.5 \%{ }^{7}$ Possible causes are injuries to the tongue, to cranial nerves VII, IX, or $\mathrm{X}$, or to the brainstem. ${ }^{6}$

This report describes a clinical case and discusses an unusual complication of facial trauma.

\footnotetext{
*Master's Degree Candidate, Department of Surgery and Integrated Clinic, Araçatuba Dental School-UNESP, Araçatuba, São Paulo, Brazil.

$\dagger$ Resident, Department of Oral Medicine and Pediatric Dentistry, State University of Londrina, Londrina, Paraná, Brazil.

$\ddagger$ Professor, Department of Oral Medicine and Pediatric Dentistry, State University of Londrina, Londrina, Paraná, Brazil.

$\S$ Professor, Department of Oral Medicine and Pediatric Dentistry, State University of Londrina, Londrina, Paraná, Brazil.
} 


\section{Report of Case}

A 32-year-old man was admitted to the Londrina State University Hospital (Londrina, Brazil) on July 14, 2013 after an automobile accident. Upon arrival at the hospital, his Glasgow scale score was 15 . He denied any addictions, allergies, comorbidities, or medication use. The neurosurgery team was responsible for the initial assessment and management because he presented with mild craniocerebral trauma, pneumoencephalos, and fractures of the C3 and $\mathrm{C} 4$ vertebrae.

An oral and maxillofacial surgery consult was requested because the patient complained of pain and difficulty bringing his teeth together. At initial evaluation by the team, he reported facial pain, anosmia, and changes in his dental occlusion. During physical examination, facial edema, bilateral periorbital ecchymosis, mild restriction in the supraversion of the right eye, epistaxis, bone misalignment in the frontozygomatic sutures and zygomaticomaxillary buttresses, anterior open bite, and maxillary mobility compatible with a Le Fort fracture were found.

Computed tomography confirmed the presence of Le Fort I and III fractures. Surgical treatment under general anesthesia was planned. After orotracheal intubation, the tube was reversed to a submental approach to allow for intermaxillary fixation during surgery. The aim of the procedure was to reduce and fix the fractures with titanium plates and screws through a bilateral vestibular sulcus approach and a coronal approach.

The patient recovered uneventfully, with proper healing of the soft tissue approaches, stable occlusion, and no esthetic compromise. After 5 months of followup, he presented an improvement of the anosmia but complained of an altered sense of taste. He reported the inability to taste chocolate, sweetened coffee, and pork. Instead of perceiving the sweet taste of sweetened coffee and chocolate or the salty taste of pork, he perceived an intense bitter taste.

The patient underwent a sequence of blind taste tests, starting with saccharose and $\mathrm{NaCl}$ solutions at a concentration of $1 \mathrm{~mol} / \mathrm{L}$, according to previous studies. ${ }^{8}$ The solutions were prepared at the State University of Londrina biochemistry laboratory and were applied at 4 specific points on the tongue (right anterior, left anterior, right posterior, and left posterior). Between applications, the patient was told to rinse his mouth with deionized water $15 \mathrm{~mL}$. He was asked to describe the flavor and intensity he perceived at each application on a scale from 1 to 9 .

Because the patient's chief complaint was related to chocolate, a second test was conducted using chocolate, in which he tasted chocolate tablets of equal size $(5 \times 5 \times 5 \mathrm{~mm})$ with different percentages of co- coa $(0,28,34,41,70$, and $85 \%)$. Between tastings, the patient rinsed twice with deionized water $15 \mathrm{~mL}$.

During the first test, he reported intensity 5 of sweet flavor to saccharose in the anterior right portion and the left front side of the tongue, intensity 4 of sweet flavor in the right rear portion, and intensity 2 of sweet flavor in the left rear portion. To $\mathrm{NaCl}$, he reported intensity 7 of bitter flavor in the right anterior part, intensity 4 of bitter flavor in left anterior part, intensity 8 of bitter flavor on the right rear part, and intensity 6 of bitter taste in the left rear part (Fig 1).

At the second test, he reported intensity 7 of bitter flavor and intensity 6 of sweet flavor for $0 \%$ cocoa; intensity 7 of bitter flavor and intensity 6 of sweet flavor for $28 \%$ cocoa; intensity 8 of bitter flavor and intensity 2 of sweet flavor for $34 \%$ cocoa; intensity 7 of bitter flavor and intensity 3 of sweet flavor for $41 \%$ cocoa; and intensity 9 of bitter flavor for $70 \%$ cocoa. When tasting $85 \%$ cocoa chocolate, the patient reported intensity 9 of bitter flavor (Table 1).

Thirty minutes after the 2 tests, the patient's tongue was anesthetized with topical anesthetic solution (10\% lidocaine) and the 2 tests were repeated. This time, to saccharose, the patient described intensity 4 of sweet flavor in the right anterior part of the tongue, intensity 3 of sweet flavor in the left anterior part, intensity 5 of sweet flavor in the right rear part, and intensity 4 of sweet flavor of in the left rear part. To $\mathrm{NaCl}$, he reported intensity 2 of salty flavor in the anterior right portion of the tongue, intensity 4 of salty flavor in the left anterior part, intensity 3 of salty flavor in the right rear part, and intensity 3 of salty flavor in the left rear part (Fig 2).

Under anesthesia, the results of the chocolate tests were intensity 3 of sweet and intensity 1 of bitter for $0 \%$ cocoa, intensity 3 of bitter and intensity 2 of sweet for $28 \%$ cocoa, intensity 3 of bitter and intensity 1 of bitter for $34 \%$ cocoa, intensity 1 of bitter and intensity 1 of sweet for $41 \%$ cocoa, intensity 2 of bitter and

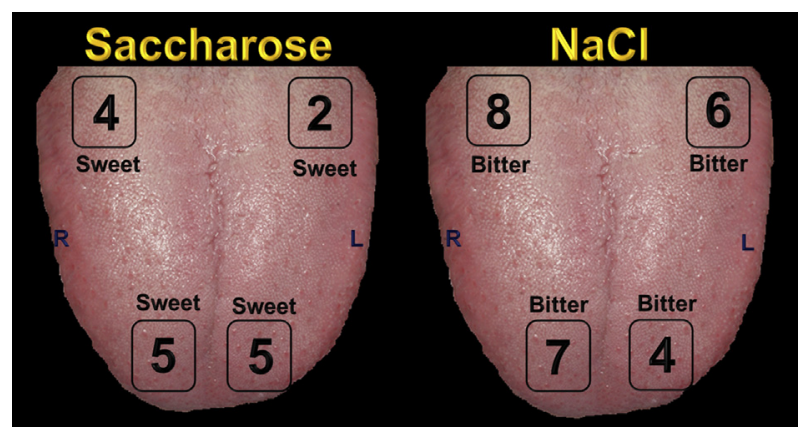

FIGURE 1. Intensity and flavor at different tongue areas, without topical anesthesia, using saccharose $1 \mathrm{~mol} / \mathrm{L}$ and $\mathrm{NaCl} 1 \mathrm{~mol} / \mathrm{L}$.

Bonardi et al. Dysgeusia From Facial Trauma. J Oral Maxillofac Surg 2016. 


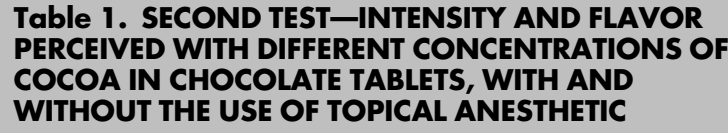

Chocolate Tasting

\begin{tabular}{lcc} 
Cocoa, & Without Topical & Topical Anesthesia \\
$\%$ & Anesthesia & With 10\% Lidocaine \\
\hline
\end{tabular}

\begin{tabular}{lll}
0 & bitter $=7$, sweet $=6$ & bitter $=1$, sweet $=3$ \\
28 & bitter $=7$, sweet $=6$ & bitter $=3$, sweet $=2$ \\
34 & bitter $=8$, sweet $=2$ & bitter $=3$, sweet $=1$ \\
41 & bitter $=7$, sweet $=3$ & bitter $=1$, sweet $=1$ \\
70 & bitter $=9$ & bitter $=2$, sweet $=1$ \\
85 & bitter $=9$ & bitter $=3$ \\
\hline
\end{tabular}

Bonardi et al. Dysgeusia From Facial Trauma. J Oral Maxillofac Surg 2016.

intensity 1 of sweet for $70 \%$ cocoa, and intensity 3 of bitter with $85 \%$ cocoa (Table 1 ).

Serum zinc and copper levels also were measured, and the results were, respectively, 105.0 and $154.14 \mathrm{~g} / \mathrm{dL}$ (reference values, 70 to $173 \mathrm{~g} / \mathrm{dL}$ for zinc and 70 to $140 \mu \mathrm{g} / \mathrm{dL}$ for copper).

\section{Discussion}

This report describes an unusual complication of facial trauma, namely partial loss of smell and taste. The patient's chief complaint after surgery was the perception of an intense bitter taste rather than sweet or salty taste when drinking sweetened coffee or eating chocolate or pork. Because the patient did not have any previous surgeries or other comorbidities, had taste buds in normal condition, and was not taking any medications, the diagnostic hypotheses were 1) a metabolic disorder caused by changes in serum levels of metals or 2) a central or peripheral nerve injury.

Laboratory tests showed a normal serum concentration of zinc and a slightly increased serum concen-

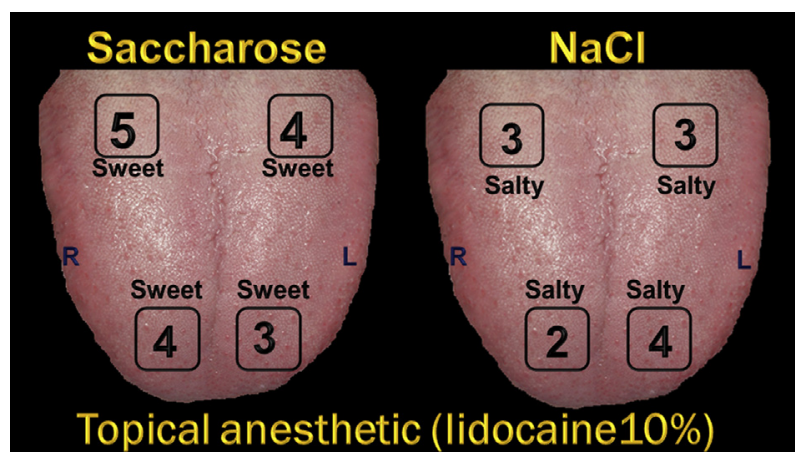

FIGURE 2. Intensity and flavor with the use of topical anesthesia using saccharose $1 \mathrm{~mol} / \mathrm{L}$ and $\mathrm{NaCl} 1 \mathrm{~mol} / \mathrm{L}$.

Bonardi et al. Dysgeusia From Facial Trauma. J Oral Maxillofac Surg 2016 tration of copper, which was not considered adequate to cause alterations of taste. Therefore, the dysgeusia was probably associated to a traumatic nerve injury.

Gustatory afferent impulses travel along cranial nerves VII, IX, and X. The gustatory fibers of cranial nerve VII are more likely to develop injuries because of the nerve's extensive and vulnerable extracranial route. ' Injury to peripheral gustatory fibers responsible for the anterior two thirds of the tongue (related to sweet and salty flavors) justifies the change in food taste reported by the patient. Because food presents chemical substances that can excite the taste buds responsible for identifying bitter and because the patient had a sensory deficit of fibers responsible for sweet and salty, the bitter flavor might have been intensified.

However, the results of the first tests showed that the patient had no dysgeusia related to sweet taste; his dysgeusia was directly related to the salty taste, was bilateral, with greater intensity on the right side, and was affecting the entire tongue. Those results suggested that trauma had not affected all flavors innervated by the tympani chorda nerve, as was supposed initially.

Taste depends on retronasal stimulus. In other words, flavor is attenuated or even eliminated without smell. ${ }^{3}$ Therefore, anosmia causes ageusia and not dysgeusia, which might explain the fact that the complaints of the patient appeared only 5 months after the surgery. The anosmia was probably causing an ageusia and masking the dysgeusia.

The literature suggests the use of topical anesthetic to differentiate dysgeusia caused by central or peripheral injuries. ${ }^{8}$ The first blind test with topical anesthetic resulted in the correction of the dysgeusia, and the second improved the correction, indicating a peripheral injury. In the 2 tests, sensory potentialization in bitter taste was observed. As described in the literature, this explains the mechanism of the topical anesthetic test of possible spontaneous hyperactivity in afferent gustative fibers. ${ }^{8}$

The patient did not present hemotympanum, hearing loss, temporal bone fractures, or facial paralysis. This suggests that a tympani chorda nerve injury inside the facial canal in the petrous part of the temporal bone was unlikely, because the tympani chorda nerve is in direct contact with the facial nerve (motor part of cranial nerve VII) inside the facial canal. ${ }^{4}$ Thus, the authors believe that the dysgeusia was caused by a tympani chorda nerve injury along its extracranial route.

An injury to the tympani chorda nerve might have occurred during the automobile accident, from deceleration forces, or during the submental reversal of the 
orotracheal tube. The submental approach was on the right side, where the dysgeusia was more severe. However, the dysgeusia was bilateral, leading to the most probable hypothesis of the dysgeusia being caused by a tympani chorda nerve injury during the automobile accident.

The treatment of dysgeusia depends on the etiology and is controversial. Zinc supplementation has been found effective in improving the gustatory sensitivity of patients with idiopathic taste disorder ${ }^{10}$; however, Nagraj et $\mathrm{al}^{11}$ in their systematic review did not find any evidence to support the role of zinc supplements for improvement in taste discrimination. In dysgeusia related to toxins or drugs, elimination of the offending agent might alleviate the problem. For traumatic injuries, there is no specific therapy available, but gradual regeneration of nerve cells can result in improvement over time. ${ }^{12}$

Studies have evaluated tympani chorda nerve alterations before and after middle ear surgery and reported taste disorders in $59.4 \%$ of cases related to unilateral section of the tympani chorda nerve, with most taste disorders ceasing spontaneously after 2 years, ${ }^{13}$ which shows a favorable prognosis and the need for follow-up.

In this case, because the dysgeusia was related to trauma, the authors opted for conservative treatment. The patient has been followed for 24 months. He has had partial remission of the dysgeusia, with a remaining complaint of some bitter taste related to salty foods.

In conclusion, post-traumatic dysgeusia is a rare complication of facial trauma. However, it is important for the maxillofacial surgeon to be aware of its possible occurrence and diagnostic methods.

\section{References}

1. Doty RL, Bromley SM: Anosmia, ageusia, and other disorders of chemosensation, in Brandt T, Caplan LR, Dichgans J, et al (eds): Neurological Disorders: Course and Treatment (ed 2). New York, NY, Elsevier Science, 2003, pp 171-183

2. Kinnamon SC, Margolskee RF: Mechanisms of taste transduction. Curr Opin Neurobiol 6:506, 1996

3. Bear MF, Connors BW, Paradiso MA: Neurociências desvendando o sistema nervoso (ed 2). Porto Alegre, Brazil, Artmed, 2002, pp 252-263

4. Alberstone CD, Benzel EC, Najm IM, et al: Bases anatômicas do diagnostico neurológico. Porto Alegre, Brazil, Artmed, 2011, pp 267-276

5. Henkin RI, Bradley DF: Hypogeusia corrected by Ni++ and Zn++. Life Sci 9:701, 1970

6. Reiter ER, DiNardo LJ, Constanzo RM: Effects of head injury on olfaction and taste. Otolaryngol Clin North Am 37:1167, 2004

7. Sumner D: Post-traumatic ageusia. Brain 90:187, 1967

8. Formaker BK, Mott AE, Frank ME: The effects of topical anesthesia on oral burning in burning mouth syndrome. Ann N Y Acad Sci 855:776, 1998

9. Costanzo RM, Becker DP: Smell and taste disorders in head injury and neurosurgery patients, in Meiselman HL, Rivlin RS (eds): Clinical Measurements of Taste and Smell. New York, NY, Macmillan, 1986, pp 565-578

10. Sakagami M, Ikeda M, Tomita H, et al: A zinc-containing compound, polaprezinc, is effective for patients with taste disorders: Randomized, double-blind, placebo-controlled, multi-center study. Acta Otolaryngol 129:1115, 2009

11. Nagraj SK, Naresh S, Srinivas K, et al: Interventions for the management of taste disturbances. Cochrane Database Syst Rev 26: CD010470, 2014

12. Mann NM: Management of smell and taste problems. Cleve Clin J Med 69:329, 2002

13. Sakagami M: Taste disturbance and its recovery after middle ear surgery. Chem Senses 30(suppl 1):i220, 2005 